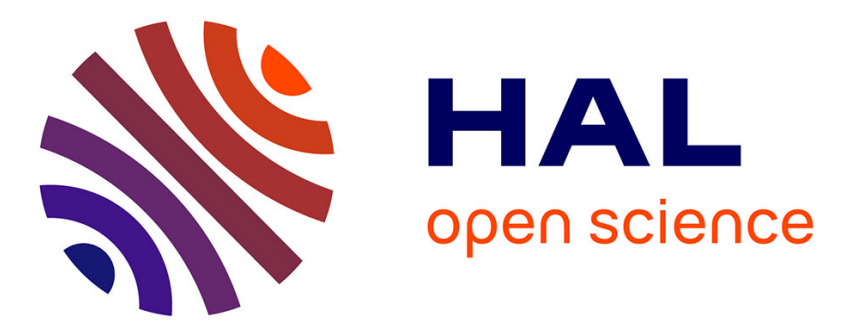

\title{
Differential effect of age on hippocampal subfields assessed using a new high-resolution 3T MR sequence.
}

Renaud La Joie, Marine Fouquet, Florence Mézenge, Brigitte Landeau, Nicolas Villain, Katell Mevel, Alice Pélerin, Francis Eustache, Béatrice Desgranges, Gaël Chételat

\section{To cite this version:}

Renaud La Joie, Marine Fouquet, Florence Mézenge, Brigitte Landeau, Nicolas Villain, et al.. Differential effect of age on hippocampal subfields assessed using a new high-resolution 3T MR sequence.. NeuroImage, 2010, 53 (2), pp.506-14. 10.1016/j.neuroimage.2010.06.024 . inserm-00538635

\section{HAL Id: inserm-00538635 https://www.hal.inserm.fr/inserm-00538635}

Submitted on 23 Nov 2010

HAL is a multi-disciplinary open access archive for the deposit and dissemination of scientific research documents, whether they are published or not. The documents may come from teaching and research institutions in France or abroad, or from public or private research centers.
L'archive ouverte pluridisciplinaire HAL, est destinée au dépôt et à la diffusion de documents scientifiques de niveau recherche, publiés ou non, émanant des établissements d'enseignement et de recherche français ou étrangers, des laboratoires publics ou privés. 


\begin{tabular}{|l|c|c|c|c|c|c|c|c|}
\hline \multirow{2}{*}{} & \multicolumn{2}{|l|}{ Whole hippocampus } & \multicolumn{2}{|c|}{ CA1 } & \multicolumn{2}{c|}{ Subiculum } & \multicolumn{2}{c|}{ Other } \\
\cline { 2 - 9 } & $\mathrm{F}$ & $\mathrm{p}$ & $\mathrm{F}$ & $\mathrm{p}$ & $\mathrm{F}$ & $\mathrm{p}$ & $\mathrm{F}$ & $\mathrm{P}$ \\
\hline Age & $\mathbf{7 . 9 0}$ & $\mathbf{0 . 0 0 7}$ & 3.52 & 0.07 & $\mathbf{2 2 . 7 9}$ & $<\mathbf{0 . 0 0 0 1}$ & 0.44 & 0.51 \\
\hline Education & 1.89 & 0.18 & 0.37 & 0.55 & 0.96 & 0.33 & 3.40 & 0.07 \\
\hline Gender & 1.62 & 0.21 & 1.30 & 0.26 & 1.42 & 0.24 & 0.63 & 0.43 \\
\hline Hemisphere & $\mathbf{2 5 . 7 1}$ & $<\mathbf{0 . 0 0 0 1}$ & $\mathbf{7 . 0 2}$ & $\mathbf{0 . 0 1}$ & $\mathbf{1 1 . 6}$ & $\mathbf{0 . 0 0 1}$ & 2.32 & 0.13 \\
\hline Age x Education & 0.22 & 0.64 & 1.00 & 0.32 & 1.47 & 0.23 & 1.67 & 0.20 \\
\hline Age x Gender & 0.01 & 0.92 & 0.75 & 0.39 & 0.94 & 0.34 & 0.01 & 0.96 \\
\hline Age x Hemisphere & 0.53 & 0.47 & 1.11 & 0.30 & $\mathbf{6 . 1 1}$ & $\mathbf{0 . 0 2}$ & 0.14 & 0.71 \\
\hline
\end{tabular}

Table 1. Results of the generalized linear mixed model assessing the effect of age, gender, education and hemisphere onto the TIV-corrected volume of the three hippocampal subparts and that of the whole hippocampus. 